\title{
Formulation and in vitro Penetration Study of Transfersomes Gel Containing Gotu Kola Leaves Extract (Centella asiatica L. Urban)
}

\author{
Silvia Surini*, Sarah, Joshita Djajadisastra \\ Faculty of Pharmacy, University of Indonesia, Depok, Jawa Barat, 16424, INDONESIA.
}

\begin{abstract}
Objective: Asiaticoside from gotu kola leaves extract (Centella asiatica L. Urban) could be used for anti-aging cosmetics. Nevertheless, its skin penetration is difficult, since it has a high molecular weight. Transfersome is a suitable carrier system that can enhance the penetration of active substances. The aims of this study were to prepare and characterize transfersomes of gotu kola leaves extract and formulated them into a gel, a control gel was also prepared without transfersome. Methods: Transfersomes were formulated with different concentrations of active substance, which was equal to asiaticoside $0.3 \%$ (F1), $0.5 \%$ (F2) and $0.7 \%$ (F3). The obtained transfersomes were evaluated for entrapment efficiency, particles morphology, particles size, polydispersity index, zeta potensial, deformability index, and penetration study using Franz diffusion cells. The best transfersomes was formulated into a gel dosage form, and then evaluated. Results: The results showed that the F1 transfersomes was the best transfersomes, hence it was incorporated into gel dosage form. The F1 transfersomes had spherical morphology with the highest entrapment efficiency of $85.80 \%$, ninety percent of the particle size distribution ( $D_{\vee} 90$ ) was below $204.3 \mathrm{~nm}$, polydispersity index of 0.125 , zeta potensial of $-36.3 \mathrm{mV}$ and deformability index of 1.12 . The cumulative amount of
\end{abstract}

penetrated asiaticoside was $1050.85 \pm 19.82 \mu \mathrm{g} / \mathrm{cm}^{2}$ for transfersomes gel and $540.21 \pm 12.28 \mu \mathrm{g} / \mathrm{cm}^{2}$ for control gel. The cumulative percentage of penetrated asiaticoside for transfersomes gel and control gel were 51.80 and $26.63 \%$, respectively. The flux of transfersomes gel containing asiaticoside and control gel were respectively 47.92 and $26.57 \mu \mathrm{g} / \mathrm{cm}^{2} / \mathrm{h}$. Conclusion: The subcutaneous penetration of asiaticoside from the transfersomes gel containing gotu kola leaves extract increased compared to the control gel without transfersomes.

Key words: Asiaticoside, Gels, Gotu kola leaves extract, In vitro penetration test, Transfersomes.

Correspondence

Dr. Silvia Surini, Faculty of Pharmacy, University of Indonesia, Depok, Jawa Barat, 16424, INDONESIA.

Phone: (021) 7270031

Email: silvia.surini@ui.ac.id

DOI: 10.5530/jyp.2018.10.8

\section{INTRODUCTION}

Centella asiatica L. Urban or commonly known as gotu kola is a medicinal plant that has been used for hundreds of years. The main content of gotu kola leaves extract is triterpenoid saponin which is commonly referred to centelloids, namely asiaticoside, madecassosid, asiatic acid, and the madecassic acid. ${ }^{1}$ Asiaticoside is a saponin compound isolated from gotu kola, has been proven to increase the synthesis of type 1 collagen in dermal fibroblasts cells through activation of TGF- $\beta .^{2}$ Asiaticoside can be used as a functional ingredient in anti-aging cosmetics. ${ }^{3}$

Asiaticoside has a large molecular weight (959.12) therefore it would be difficult to penetrate through the skin. ${ }^{4,5,6}$ To give a functional effect in anti-aging cosmetic, it needs a carrier, transfersomes which can penetrate through the skin barrier of stratum corneum. ${ }^{7}$ Transfersome is the improvement of liposomes and recognized more effective in delivering active substances through the skin compared to liposomes, because of its flexible ability to be deformed when passing through small pores in the skin, which is advantageous in increasing the absorption of active ingredient. ${ }^{7,8}$

Transfersome formulation can be applied into a semisolid dosage form, in this case gel formulation was chosen because it has a high-water content so it can hydrate the skin and easily spreads when applied., ${ }^{9} 10$

The aims of this study were to prepare and to characterize transfersomes of gotu kola leaves extract and formulated transfersomes into gel, compared to control gel. The gels were evaluated for entrapment efficiency, particles morphology, particles size, polydispersity index, zeta potential, deformability index, and penetration study using Franz diffusion cells.
Thin layer chromatography (TLC) densitometry was used to analyze the level of active substances contained in the extract.

\section{MATERIALS AND METHODS}

\section{Materials}

Gotu kola leaves extract (Centella asiatica L. Urban) (Changsha Vigorous Tech, China), asiaticoside (Baoji Guokang Bio Technology, China), Phospolipon 90G (Donated by GMBH, Germany), ethanol (Merck, Germany), methanol (Merck, Germany), chloroform (Merck, Germany), dichlorometane (Merck, Germany), anhydrous acetic acid (Merck, Germany), sulphuric acid (Merck, Germany), sodium hydroxide (Merck, Germany), propylene glycol (Dow Chemical Co.), potassium dihydrogen phosphate (Merck, Germany), triethanolamin (Croda, Singapore), demineralized water (Brataco, Indonesia), female Sprague Dawley mice skin aged 2-3 month weighed 200 - 250 gr (Institute Pertanian Bogor, Indonesia)

\section{METHODS}

\section{Determination of asiaticoside levels in gotu kola leaves extract by thin layer chromatography (TLC) densitometry}

The standard solution for calibration curve were $1000 \mu \mathrm{g} / \mathrm{mL}, 800 \mu \mathrm{g} / \mathrm{mL}$, $600 \mu \mathrm{g} / \mathrm{mL}, 500 \mu \mathrm{g} / \mathrm{mL}, 400 \mu \mathrm{g} / \mathrm{mL}, 200 \mu \mathrm{g} / \mathrm{mL}$, and $100 \mu \mathrm{g} / \mathrm{mL}$. Each $2 \mu \mathrm{L}$ of standard solutions and the sample solution were spotted on the 
plates of silica gel $\mathrm{F}_{254}$ and put into the mobile phase mixture of chloroform-methanol-water 60: 25: $4(\mathrm{v} / \mathrm{v})$ until the mobile phase front was $1 \mathrm{~cm}$ from the edge of the plate, then it was sprayed with reagent Liebermann-Burchard, heated at $100^{\circ} \mathrm{C}$ for $10 \mathrm{~min}$. The plates that spotted then immediately analyzed using TLC scanner at optimum wavelengths of $506 \mathrm{~nm} .^{11,12,13}$

\section{Transfersomes formulation}

The transfersomes of gotu kola leaves extract were prepared by thin layer hydration method and the trasfersomes composition is showed at Table 1. Phospholipid was dissolved in dichloromethane and put in a round bottom flask. Then the solution was evaporated using a rotary evaporator vacuum in that round bottom flask with a temperature of $37 \pm 2^{\circ} \mathrm{C}$. The initial velocity used was 50 rotations per $\min (\mathrm{rpm})$ then it was increase to $25 \mathrm{rpm}$ every $2 \mathrm{~min}$ until reached $150 \mathrm{rpm}$. Once all the solvent evaporates and formed a thin layer on the inner wall of the flask, nitrogen gas was flowed. Thin layer formed was then stored for $24 \mathrm{~h}$ in the refrigerator.

After the thin layer was formed, the hydration process was then performed using a mixture of gotu kola leaves extract, Tween 80, and phosphate buffer saline $\mathrm{pH} 7.4$ until all the thin layer was peeled off using the glass beads. Hydration process was carried out at the temperature of $37 \pm 2^{\circ} \mathrm{C}, 100 \mathrm{rpm}$ for $30 \mathrm{~min}$ and without the vacuum condition. After the thin layer was fully hydrated, the transfersomes suspension were collected in vial then the particle size of the vesicle was reduced by ultrasonification for $5 \mathrm{~min}$ with the amplitude of 25 . Then the transfersomes suspension was stored in a refrigerator. ${ }^{14,15}$

\section{Characterization of transfersomes Entrapment efficiency}

The entrapment efficiency was determined as the percentage entrapment of the drug added, by separating the unentrapped drug using centrifugation method. Transfersomes of gotu kola leaves extract was taken as much as $1.0 \mathrm{~mL}$ and centrifuged at $13.000 \mathrm{rpm}$ for 2 times for 1 hour each. Transfersomes will separate into a precipitate and supernatant. The supernatant was discarded, the precipitate was collected, and then disrupted using methanol Then, the entrapment efficiency was expressed as: ${ }^{17}$

$\%$ Entrapment of efficiency $=\frac{\text { Experimental asiaticoside content }}{\text { Theoritical asiaticoside content }} \times 100 \%$

\section{Transfersomes morphology}

The transfersomes morphology was observed by using Transmission Electron Microscope (TEM). The sample was stained by placing a drop of transfersomes suspension on a carbon coated grid. Then, a drop of $1 \%$ phosphotungstic acid was placed on the grid. The excess was removed, and the samples were examined by TEM at 20 and $25 \mathrm{kV} .^{15}$

\section{Particle size distribution and zeta potential characterization}

Particle size distribution and zeta potential can be determined using particle size analyzer (PSA) with dynamic light scattering (DLS) method. A drop of sample was dissolved in $10 \mathrm{~mL}$ of phosphate buffer saline at pH 7.4 then, size measurement was done by using Malvern particle size analyzer instrument. ${ }^{16}$

\section{Degree of deformability}

Deformability index was measured by extrusion. Transfersome suspension was passed through a $100 \mathrm{~nm}$ sized polycarbonate membrane. The amount of transfersomes suspension was extruded for five min, then the vesicle size was measured using dynamic light scattering method before and after filtration. The degree of deformability was calculated using the following formula: ${ }^{7}$

$$
\mathrm{D}=\mathrm{J}\left(\frac{\mathrm{r}_{\mathrm{v}}}{\mathrm{r}_{\mathrm{p}}}\right)^{2}
$$

where, $D$ is the deformability of vesicle membrane, $J$ is the amount of suspension passed in $5 \mathrm{~min}, r v$ is the size of vesicle (after passed) and $r p$ is the pore size of the barrier. $^{2}$

$$
\mathrm{D}=\mathrm{J}\left(\frac{\mathrm{r}_{\mathrm{v}}}{\mathrm{r}_{\mathrm{p}}}\right)^{2}
$$

\section{Gel Formulation}

The composition of gel formulation containing gotu kola leaves extract was described at Table 2. Gel preparation was start by dispersing Carbopol 940 into a distilled water then kept it for $24 \mathrm{~h}$. Then, the Carbopol gel base was homogenized using a homogenizer at $1500 \mathrm{rpm}$ until the Carbopol base gel formed. Then, triethanolamine and propylene glycol was added into the gel base. Once the gel formed, the suspension of transfersomes was added into F1 and gotu kola leaves extract solution was added to F2. Both gels were homogenized using a homogenizer at stirring speed of $500 \mathrm{rpm}$ for $15 \mathrm{~min}$ and the gels were evaluated for organoleptic, homogeneity, $\mathrm{pH}$ measurement, and viscosity and rheology.

\section{In vitro Penetration Test ${ }^{18,19}$}

The membrane used was the abdominal skin of female Sprague Dawley rats aged 8-10 weeks with the weight of \pm 200 gs. The test was performed using Franz diffusion cells with the diffusion area of $2.0096 \mathrm{~cm}^{2}$ and the volume of compartment was $15 \mathrm{~mL}$. The receptor compartment filled with a mixture of $70 \%$ ethanol solution and phosphate buffer saline at $\mathrm{pH} 7.4$ which kept in the temperature about $37 \pm 0.5^{\circ} \mathrm{C}$ and stirred with a magnetic stirrer at a speed of $250 \mathrm{rpm}$. After that, the abdominal skin of mice was placed between the donor compartment with the receptor compartment to the position of the stratum corneum facing upwards. A sample of $2 \mathrm{~g}$ was applied to the skin surface with an area of diffusion was $2.0096 \mathrm{~cm}^{2}$. The experiment lasted for $24 \mathrm{~h}$. The amount of asiaticoside penetrated through the membrane was determined by TLC densitometry.

\section{RESULTS}

\section{Determination of Asiaticoside Levels in Gotu Kola Leaves Extract by Thin Layer Chromatography (TLC) Densitometry}

In this research, the amount of asiaticoside contained in gotu kola leaves extract was measured using Thin Layer Chromatography Densitometry. The results showed that the asiaticoside concentration in the gotu kola leaves extract was $23.14 \pm 0.41 \%$.

\section{Transfersomes Characterization \\ Determination of Entrapment Efficiency}

Entrapment efficiency was determined by separating the unentrapped drug using centrifugation method. From the calculation of the entrapment efficiency it was obtained an average percentage of entrapment for the F1, F2 and F3 were $85.80 \pm 0.22 \% ; 56.90 \pm 0.77 \%$; and $41.67 \pm 0.32 \%$, respectively. The absolute value of the extract entrapped in transfersomes of F1, F2 and F3, which were $278 \pm 0.73 \mathrm{mg} ; 307.39 \pm 4.17 \mathrm{mg}$; and $315.33 \pm 2.47 \mathrm{mg}$. 


\section{Transfersom Morphology Vesicle}

The vesicles morphology of transfersomes was observed using Transmission Electron Microscopy (TEM). The transfersome micrograph is shown at Figure 2, all formula (F1, F2, F3) of the gotu kola leaves extract transfersomes resulted the spherical transfersomes without pores.

\section{Particle Size Distribution, Polydispersity Index and Zeta Potential}

In this study, particle size distribution and zeta potential were analyzed by particle size analyzer with the method of Dynamic Light Scattering (DLS). Figure 1 showed the particle size distribution of the transfersomes of gotu kola leaves extract, and the measurement of the transfersomes particle size was showed at Table 3 in the specifications of $\mathrm{D}_{\mathrm{v}} 10, \mathrm{D}_{\mathrm{v}} 50$, $\mathrm{D}_{\mathrm{v}} 90$, and $\mathrm{D}_{\mathrm{v}}$ mean. DLS can determine particle size which followed by the value of polydispersity index (PDI). Polydispersity index value of F1, F2, F3 transfersomes was $0.125 \pm 0.008,0.256 \pm 0.053$, and $1.000 \pm 0,000$, respectively. The measurement of zeta potential was done using the same tools on the transfersomes suspension. The results showed that the value of zeta potential of $\mathrm{F} 1, \mathrm{~F} 2$ and $\mathrm{F} 3$ transfersomes were $-36.3 \mathrm{mV} \pm 0.30$, $-32,2 \pm 1.32 \mathrm{mV}$ and $-20.63 \mathrm{mV} \pm 0.40$, respectively. Table 4 displays the value of PDI and zeta potential of the transfersomes.

\section{Deformability Index Test}

Deformability index was used to test the flexibility of transfersom. ${ }^{16}$ The results of the deformability index measurement were shown at Table 4. It shows that F1 has the lowest deformability index.

\section{In vitro Penetration Test}

Figure 3 shows the cumulative amount of the penetrated asiaticoside from the transfersomes gel and the control gel (without transfersomes) through the rat skin membrane for $24 \mathrm{~h}$, which were $1050.85 \pm 19.82$ $\mathrm{ug} / \mathrm{cm}^{2}$ and $540.21 \pm 12.28 \mathrm{ug} / \mathrm{cm}^{2}$, respectively. The amount of asiaticoside penetrated from transfersomes gel and control gel respectively were $51.80 \% \pm 0.97$ and $26.63 \pm 0.60 \%$. Flux value at a steady state for transfersomes gel and control gel respectively were $47.92 \pm 1.74 \mathrm{mg} / \mathrm{cm}^{2} /$ hour and $26.57 \pm 0.77 \mathrm{mg} / \mathrm{cm}^{2} /$ hour.

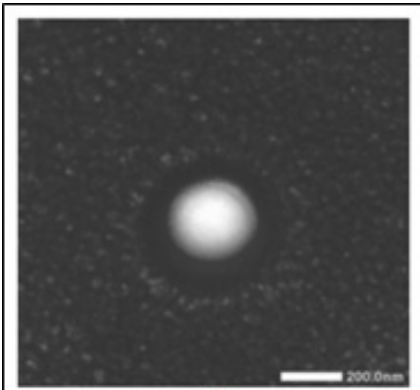

(F1)

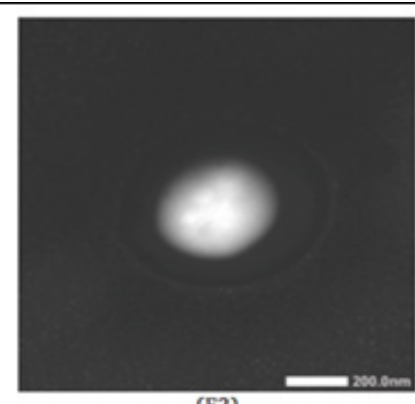

(F2)

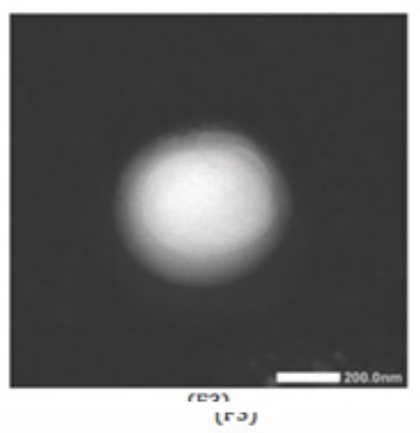

Figure 1: The micrographs of the gotu kola leaves extract transfersomes

\section{DISCUSSION}

In this study, the transfersomes of gotu kola leaves extract were prepared by thin layer hydration method. Then, the entrapment efficiency is determined as the percentage entrapment of the drug entrapped. Among the other formulas, F1 showed the highest value of entrapment efficiency $(85 \%)$, since $\mathrm{F} 1$ was produced with the highest ratio of lipid (13x). Besides that, the rest of the extract was not entrapped, it presumably because the rest of the extract was degraded during the manufacturing process or analyzes process. But there is no supporting data to prove it because the concentration of extract that is not entrapped are not calculated. On the other hand, F3 transfersomes, which were the smallest ratio of lipid (5.7x), had the lowest value of entrapment efficiency. It suggests that the extract cannot be fully entrapped, since the transfersomes was lack of the former lipid.

The particle size was an important parameter in the preparation of nanoparticles, such transfersomes. Parameter of particle size distribution measurement used were $d v$ or diameter based on volume, because it can describe heterogeneity of particle size of the sample. ${ }^{20}$ Figure 1 displays the particle size distribution curves of the microcapsules formulas. The results of the measurement of particle size distribution of all formulas shows the sequence from the smallest particle size was $\mathrm{F} 1<\mathrm{F} 2<\mathrm{F} 3<\mathrm{F} 4$ (Table 3). It reveals that $\mathrm{F} 1$ transfersomes have the smallest $\mathrm{D}_{\mathrm{v}} 90$, which is $204.3 \mathrm{~nm}$. It suggests that the concentration of gotu kola leaves extract in the transfersomes affected the particle size, the higher the concentration of gotu kola leaves extract, the bigger the transfersome particle size.

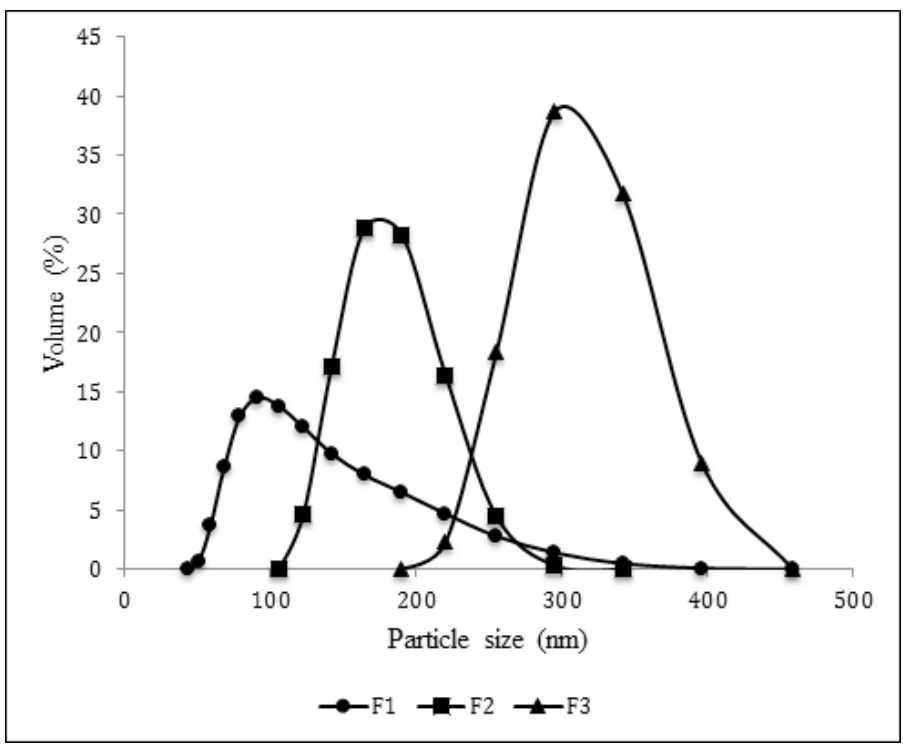

Figure 2: Particle size distribution of the gotu kola leaves extract transfersomes

Table 1: Formulation of gotu kola leaves extract transfersomes.

\begin{tabular}{cccc}
\hline \multirow{2}{*}{ Materials } & \multicolumn{3}{c}{ Concentration (\%) (b/v) } \\
\cline { 2 - 4 } & F1 & F2 & F3 \\
\hline $\begin{array}{c}\text { Gotu kola leaves extract } \\
\text { equivalent to asiaticoside }\end{array}$ & 0.3 & 0.5 & 0.7 \\
Phospholipon 90G & 4 & 4 & 4 \\
Tween 80 & 0.7 & 0.7 & 0.7 \\
Phosphate buffer saline at pH 7,4 & Ad 100 & Ad 100 & Ad 100 \\
\hline
\end{tabular}






\begin{tabular}{ccc} 
Table 2: Gel formulation of gotu kola leaves extract transfersomes. \\
\hline Materials & \multicolumn{2}{c}{ Concentration (\%) } \\
\cline { 2 - 3 } & G1 & G2 \\
\hline Carbopol 940 & 1 & 1 \\
Triethanolamin & 0,5 & 0,5 \\
Propylene glycol & 12,5 & 12,5 \\
Transfersomes of gotu kola leaves \\
extract equivalent to asiaticoside \\
$\begin{array}{c}\text { Solution of gotu kola leaves extract } \\
\text { equivalent to asiaticoside } \\
\text { Demineralized water }\end{array}$ & 0,2 & - \\
\hline
\end{tabular}

Figure 3: The cumulative amount of the penetrated asiaticoside from the transfersomes gel $(\bullet)$ and control gel $(\bullet)$.

Table 3: The of particle size measurement of the gotu kola leaves extract transfersomes.
\begin{tabular}{ccccc}
\hline Formula & $\mathrm{D}_{\mathrm{v}-10}(\mathrm{~nm})$ & $\mathrm{D}_{\mathrm{v}-50}(\mathrm{~nm})$ & $\mathrm{D}_{\mathrm{v}-90}(\mathrm{~nm})$ & $\mathrm{D}_{\mathrm{v}-\mathrm{mean}}(\mathrm{nm})$ \\
\hline $\mathrm{F} 1$ & 69.8 & 109.3 & 204.3 & 124.6 \\
F2 & 136.3 & 177.0 & 229.7 & 179.5 \\
F3 & 250.3 & 308.7 & 381.3 & 308.4
\end{tabular}

\begin{tabular}{cccccc}
\multicolumn{6}{l}{ Table 4: The characterizations of the gotu kola leaves extract transfersomes } \\
\hline Transfersomes & Vesicle Morphology & Polydispersity Index & Deformability Index & Zeta Potential (mV) & Entrapment Efficiency (\%) \\
\hline F1 & Spheris & $0.125 \pm 0.008$ & 1.12 & $-36.30 \pm 0.30$ & $85.80 \pm 0.22$ \\
F2 & Spheris & $0.256 \pm 0.053$ & 2.34 & $-32.20 \pm 1.32$ & $56.90 \pm 0.77$ \\
F3 & Spheris & $1.000 \pm 0.000$ & 2.64 & $-20.63 \pm 0.40$ & $41.67 \pm 0.32$ \\
\hline
\end{tabular}

Moreover, DLS can determine PDI value, which is range from 0 to 0.6 . If the value of PDI $>0.6$ indicates that the sample has a very broad size distribution. The smaller value of PDI (near zero) illustrating that the size of distribution is more homogeneous particle size distribution..$^{22}$ It can be seen in Table 3 and Table 4 that PDI of each formula increases with increasing particle size. The highest polydispersity index value of F3 was caused by the large particles which were prone to aggregation. ${ }^{20}$

In addition to particle size distribution and polydispersity index (PDI), the measurement of zeta potential was done using the same tools on the transfersomes suspension. Zeta potential is a measure of the magnitude of the electrostatic potential or repulsive force among the same electrical charge of particles in suspension. Zeta potential is an important parameter to describe the stability of the dispersion system. Zeta potential can give an idea that particles in suspension undergo aggregation or flocculation. ${ }^{21}$ A sample can be said to be stable if it has a value of zeta potential is more positive than of $+30 \mathrm{mV}$ or more negative than $-30 \mathrm{mV}$. The results revealed that the $\mathrm{F} 1$ and $\mathrm{F} 2$ transfersomes were more stable than the F3, because their zeta potential is more than $30 \mathrm{mV}$. Negative value of zeta potential might be getting from the lipid composition in the formula. Phosphatidylcholine is a zwitterionic compound with the isoelectric point between 6 and 7. During the process of formulation phosphate buffer saline at $\mathrm{pH} 7.4$ was used as the hydrating medium, where the $\mathrm{pH}$ was a little bit higher than the isoelectric point of phosphatidylcholine, causing phosphatidylcholine carried a negative charge. ${ }^{22}$
Deformability index is unique and important parameter in transfersomes preparation. These parameters that distinguish transfersomes with another vesicle systems. Deformability index was used to examine the flexibility of transfersom. ${ }^{16}$ The result shows that F1 transfersomes has the lowest deformability index. However, these results did not show any significant value because of the membrane pore size that is used does not vary much with particle sizes in the tested suspension. Deformability index value is influenced by the concentration of phospholipid and surfactant used. The use of excessive surfactant can lower deformability index value as it can lead to the formation of micelles. The larger the index value of deformability, then the transfersomes will be more flexible and allows transfersomes to penetrate through the skin pores smaller than the size transfersomes itself. ${ }^{14}$

Based on the characterization results of transfersomes that has been done, we can determine the best transfersomes formulation chosen for further formulated into a gel formulation. Formula selected was a formula with the highest percentage of drug entrapped, had spherical morphology, the value of polydispersity index was close to 0 and had the value of zeta potential which more positive than $+30 \mathrm{mV}$ or more negative than $-30 \mathrm{mV}$. Based on the summary results shown in the Table 4, F1 transfersomes was chosen because it had a spherical shape, the highest percentage of drug entrapped $(85.80 \pm 0.22 \%)$, polydispersity index of $0.125 \pm 0.008$, zeta potential value of $-36.3 \pm 0.30 \mathrm{mV}, \mathrm{D}_{\mathrm{v}} 90$ particle size of $204.3 \mathrm{~nm}$, 
and deformability index of 1.12. Therefore, it can be decided that F1 was the best formula compared to F2 and F3, and selected for gel formulation. The penetration study was done during $24 \mathrm{~h}$ with sampling at 17 -point intervals. During the test of each volume samples taken were immediately replaced with the same volume of the medium to keep the concentration of drug in the receptor compartment remain constant, forming sinks conditions that will make a difference to the concentration gradient between the donor compartment and the receptor compartment. ${ }^{23}$

Based on the result, it reveals that the absorption process of asiaticoside from transfersomes gel had a shorter lag time than control gel. Flux each time obtained from the comparison between the cumulative number of penetrated versus time. ${ }^{23}$ There are several factors related to the physicochemical properties that can affect the ability of drug penetration into the skin. Appropriate molecular weight drug molecules that can penetrate is less than $400 .{ }^{4}$ Asiaticoside has a molecular weight of 959.12. ${ }^{12}$ Active substances with small molecular weight can penetrate faster as compared to the active substances with a high molecular weight. ${ }^{24}$

In addition, the physicochemical factors that can affect the penetration is the partition coefficient of the drug in the n-octanol-water. Partition coefficient values appropriate to be able to penetrate is -1 to $4 .{ }^{4}$ Asiaticoside have a partition coefficient of $0.908 .^{25}$ The higher the value of the partition coefficient, the greater the penetration of the drug. ${ }^{26}$ That is why asiaticoside in control gel was not easy to penetrate. Penetration test results conducted on transfersomes gel and control gel showed a significant difference, the penetration of transfersomes gel was two times higher than the control gel. This proves that with the transfersomes formulation able to increase the penetration of active substances. Particle size reduction in transfersomes shown to increase the penetration of asiaticoside. Transfersomes contain surfactants that serves as edge activator in transfersomes functioned to destabilize the vesicles and increase the deformability of the phospholipid bilayer by lowering the interfacial tension so that the vesicle transfersomes can carry drugs through the pores smaller than the size of the drug itself..$^{27,28}$

\section{CONCLUSION}

Gotu kola leaves extract was formulated into transfersomes vesicles with the best formula was F1, which had a spherical shape, the highest entrapment efficiency of $85.80 \pm 0.22 \%, D_{\mathrm{V}} 90$ of $204.3 \mathrm{~nm}$, polydispersity index value of $0.125 \pm 0.008$, zeta potential of $-36.3 \pm 0.3 \mathrm{mV}$ and deformability index of 1.12 . The subcutaneous penetration of asiaticoside from the transfersomes gel containing gotu kola leaves extract increased compared to the control gel without transfersomes.

\section{ACKNOWLEDGMENT}

The authors gratefully acknowledge to Directorate of Research and Community Engagements of Universitas Indonesia for financial support.

\section{CONFLICT OF INTEREST}

The authors have no conflict of interest to declare.

\section{ABBREVIATION USED}

TGF-b: transforming growth factor beta; TLC: Thin layer chromatography; TEM: transmission electron microscope; DLS: dynamic light scattering; PDI: Polydispersity index.

\section{REFERENCES}

1. Cevc G, Schätzlein A, Blume G. Transdermal drug carriers: Basic properties, optimization and transfer efficiency in the case of epicutaneously applied peptides. J. Control. Release. 1995;36(1-2):3-16.

2. Lee J, Jung E, Kim Y, Park J, Park J, Hong S, Park D. Asiaticoside induces human collagen I synthesis through TGFbeta receptor I kinase (TbetaRI kinase)independent Smad signaling. Planta Med. 2006;72(4): 324-8.

3. Yasurin P. Review: The Bioavailability Activity of Centella asiatica. 2016;1-9.

4. Chandrashekar NS, Rani RHS. Physicochemical and pharmacokinetic parameters in drug selection and loading for transdermal drug delivery. IJPER. 2008; 70(1):94-6.

5. Chen J, Lu L, Gao S, Lin H, Wei S, Zhang Y, Gu J. Liposome containing asiaticoside and the use thereof. United States Patent Application Publication. 2006;1(13):19-22.

6. Kim WJ, Kim JD, Veriansyah B, Kim J, Oh SG, Raymond R. Extraction of asiaticoside from Centella asiatica: Effects of solvents and extraction methods. Proceeding of KIChE Fall National Meeting, Korea. 2007;134.

7. Widyanati P, Jufri M, Elya B. Formulation and penetration study of liposome gel xanthone of extract mangosteen. Int. J. Pharm. Sci. Rev. Res. 2014;27(1):1-6.

8. Katz LM, Dewan K, Bronaugh RL. Nanotechnology in cosmetics. Food Chem Toxicol. 2015;85:127-37.

9. Baki G, Alexander KS. Introduction to Cosmetic Formulation and Technology. Wiley; 2015

10. Shaji J, Lal M. Preparation, optimization and evaluation of transfersomal formulation for enhanced transdermal delivery of a cox-2 inhibitor. 2014;6(1):467-77.

11. Ganse H, Gbaguidi F, Agbani P, Josse R, Moudachirou M, Sinsin B, et al. Densitometric HPTLC quantification of asiaticoside isolated from Centella asiatica(L.) Urb (Apiaceae) of Benin. IJBCS. 2010;4(4):857-63.

12. James J, Dubery I. Identification and quantification of triterpenoid centelloids in Centella asiatica(L.) Urban by densitometric TLC. Journal of Planar Chromatography - Modern TLC. 2011;24(1):82-7.

13. Pramono S, Ajiastuti D. Standardization of Centella asiatica(L.) Urban herbs extracts based on ectatikoside content in KLT-densitometry. Majalah Farmasi Indonesia. 2004;15(3):118-23.

14. Chaudhary H, Kohli K, Kumar V. Nano-transfersomes as a novel carrier for transdermal delivery. Int. J. Pharm. 2013;454(1):367-80.

15. El Zaafarany GM, Awad GAS, Holayel SM, Mortada ND. Role of edge activators and surface charge in developing Ultradeformable vesicles with enhanced skin delivery. Int. J. Pharm. 2010;397(1-2):164-72

16. Jadupati M, Amites G, Kumar NA. Transfersome: An opportunistic carrier for transdermal drug delivery system. IRJP. 2012;3(3):35-8.

17. Walve JR, Bakliwal SR, Rane BR, Pawar SP. Transfersomes: A surrogated carrier for transdermal drug delivery system. Int J Appl Biol Pharm. 2011;2(1):204-13.

18. Barbara WK, William GR. Methods for Skin Absorption. CRC Press; 1990.

19. Paolino D, Cosco D, Cilurzo F, Trapasso E, Morittu VM, Celia C, et al. Improved in vitro and in vivo collagen biosynthesis by asiaticoside-loaded ultradeformable vesicles. J. Control. Release. 2012;162(1):143-51.

20. Malvern. A Basic Guide to Particle Characterization. United Kingdom: Malvern; 2012

21. Malvern. Dynamic Light Scattering, 1-6. Uniteed Kingdom: Malvern; 2011.

22. Duangjit $S$, Opanasopit $P$, Rojanarata T, Ngawhirunpat T. Characterization and in vitro skin permeation of meloxicam-loaded liposomes versus transfersomes. J Drug Deliv. 2011;418316.

23. Sinko JP, Martin NA. Martins Physical Pharmacy and Pharmaceutical. Lippincott Williams and Wilkins, wolter Kluwer. 2006;6.

24. Barrett CW. Skin penetration. J Cosmet Sci. 1969;20:487-99.

25. Yang-Yang Z, Li C, Li Z, Ling Z, Xiao-chu C. Determination of equilibrium solubility and apparent oil/water partion coefficient of asiaticoside. Pharmaceutical Journal of Chinese People's Liberation Army. 2013;03

26. Stafford RG, Mehta M, Kemppainen BW. Comparison of the partition coefficient and skin penetration of a marine algal toxin (lyngbyatoxin A). Food Chem Toxicol. 1992;30(9):795-801.

27. Jacob L, KR A. A Review on Surfactants as Edge Activators in Ultradeformable Vesicles for Enhanced Skin Delivery. IJBPS. 2013;4(3):337-44.

28. Miatmoko A, Kuwano K, HattoriY, MaitaniY, Yonemochi E. Evaluation of transfer some and protransfersome for percutaneous delivery of cisplatin in hairless mice. J. Pharm. Pharmacol. 2015;5(1):1-7. 